SCIENTIFIC LETTER

\title{
Three dimensional segmented myocardial perfusion images by selective intracoronary injection of contrast using 256 slice cone beam computed tomography
}

\author{
N Funabashi, K Yoshida, H Tadokoro, K Nakagawa, N Komiyama, K Odaka, T Tsunoo, S Mori, \\ M Endo, S Tanada, I Komuro
}

Heart 2005;91:1349-1351. doi: 10.1136/hrt.2004.045997

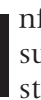
nformation of the segmented left ventricular (LV) area supplied by every coronary artery is potentially useful for strategy and practice of percutaneous coronary intervention, coronary artery bypass surgery, or percutaneous transluminal septal myocardial ablation (PTSMA). ${ }^{12}$

Myocardial contrast echocardiography (MCE) with intracoronary contrast injection has been used to evaluate the segmented LV area supplied by a coronary artery but it is invasive and evaluation of myocardial enhancement by ultrasound contrast material may depend upon the skill of the investigator. ${ }^{1}$

With a prototype high speed cone beam computed tomography (CT) apparatus employing 256 detector rows (Athena, Sony-Toshiba), an entire heart can be imaged within a single gantry rotation. ${ }^{34}$ Using this technique, combined with selective intracoronary injection of contrast, we determined the segmented LV area supplied by every coronary artery selectively.

\section{METHODS}

Two domestic pigs (20 kg each) were anaesthetised with isoflurane, and catheters positioned in the left anterior descending branch (LAD) of the coronary artery in pig 1 and the left circumflex branch (LCX) in pig 2, via the femoral arteries. The heart rate of pigs ranged between $70-80$ beats per minute.

Scan conditions for the 256 slice cone beam CT were: $120 \mathrm{kV}, 200 \mathrm{~mA}, 1.0$ second exposure, 1.0 second gantry rotation time, $0.5 \mathrm{~mm}$ slice thickness, and $256 \times 0.5 \mathrm{~mm}$ slice collimation. For comparison, those for the 16 slice multislice CT scanner routinely used at our institute (SOMATOM Sensation 16, Siemens) were: $120 \mathrm{kV}, 250 \mathrm{~mA}, 0.42$ second exposure, 0.42 second gantry rotation time, $0.75 \mathrm{~mm}$ slice thickness, and $16 \times 0.75 \mathrm{~mm}$ slice collimation.

To begin, $10 \mathrm{ml}$ of the iodinated contrast material (300 mgI/ml) diluted with $40 \mathrm{ml}$ of saline was injected at a rate of $3 \mathrm{ml} / \mathrm{second}$.

Entire heart scanning and injection of contrast started simultaneously and the scanning was continued for 25 seconds. The radiation dose was $100 \mathrm{mSV}$ for 25 seconds. ${ }^{5}$

Reconstruction parameters were a voxel size of $0.468 \times 0.468 \times 0.500 \mathrm{~mm}^{3}$ with $0.500 \mathrm{~mm}$ reconstruction increment along the $\mathrm{z}$ axis and a matrix size of $512 \times 512 \times 256$. Convolution kernels were standard body kernels; FC10.

The temporal resolution of this new CT is 1 second and spatial resolution was approximately $1.0 \mathrm{~mm}$ (transverse) and $1.3 \mathrm{~mm}$ (longitudinal).

The reconstruction was performed at every 0.1 second interval.

\section{RESULTS}

At 2 seconds, only the coronary arterial trees of LAD (pig l) or LCX (pig 2) could be observed without myocardial enhancement. At 5 seconds, segmented myocardial enhancement of LAD and LCX could be observed with the coronary arterial trees.

We selected the most static images of the LV at around 5 seconds after contrast injection, so as to avoid any gaps from cardiac motion artefact. Axial source images clearly revealed segmented LV myocardial enhancement of the anterior and apical wall and interventricular septum (IVS) in pig 1 and the lateral and posterior wall in pig 2. Volume rendered images from the anterior and the left anterior views revealed only the anterior and apical wall and IVS portion of the LV myocardium supplied exclusively by the LAD in pig 1 and the lateral and posterior wall of LV myocardium supplied by the LCx in pig 2, together with the coronary artery (fig 1); the segmented LV area could be easily recognised three dimensionally.

\section{DISCUSSION}

A prototype 256 slice cone beam CT provides complete volumetric data within a single gantry rotation. In this CT, by combination of reconstruction technique, it is possible to obtain a configuration of the heart without any gaps caused by cardiac motion artefacts. This new prototype CT has a unique character, synchrony, for the acquisition of the pulsating heart. The scanning mechanism can accommodate a rotation speed of up to 0.5 second/rotation and ECG gated acquisition will be possible in the next generation scanner.

In this study using small pigs, we demonstrated segmented LV perfusion combined with selective coronary arterial injection and we successfully observed the segment LV enhancement three dimensionally.

Two plans can be envisaged for the future. Firstly, the combination of a selective angiography system with the CT system in which the CT acquisition with selective coronary artery injection can be achieved without any movement of the patient. In fact, this angiography system with multislice CT is already in use. Using the new system in which an angiography system is combined with a new 256 slice CT, it is possible to obtain the same segmented LV enhancement.

Secondly, acquisition using the new 256 slice CT combined with intravenous injection non-invasively. By this method, even though the segmentation of LV myocardial enhancement cannot be performed, we can evaluate the three

Abbreviations: $\mathrm{CT}$, computed tomography; IVS, interventricular septum; LAD, left anterior descending; LCx, left circumflex; LV, left ventricular; MCE, myocardial contrast echocardiography; PTSMA, percutaneous transluminal septal myocardial ablation 


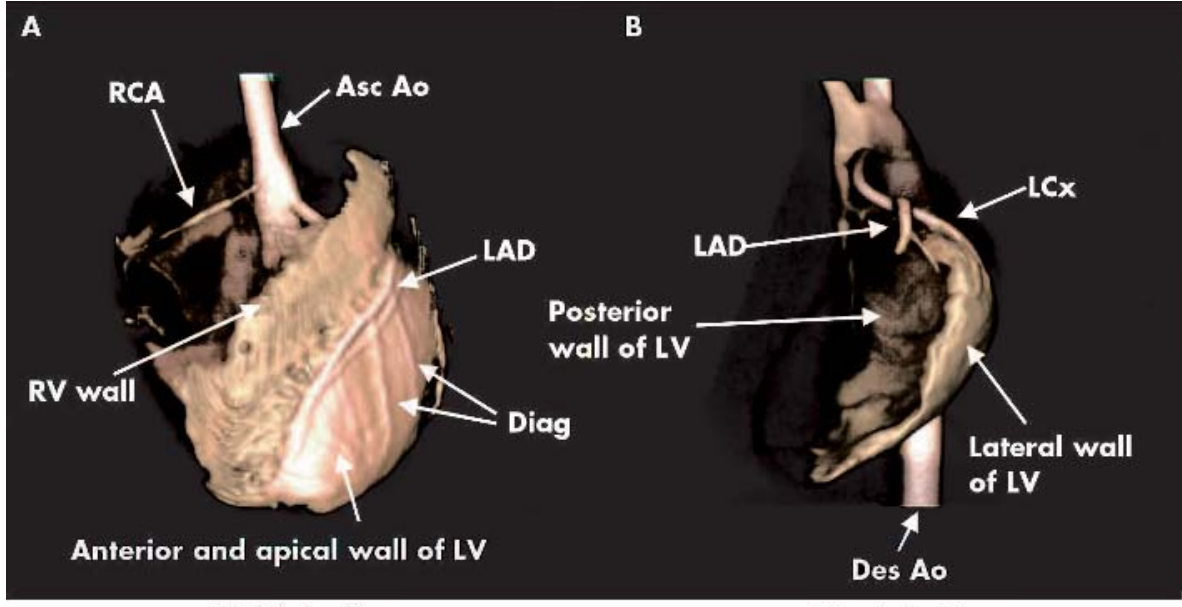

LAD injection

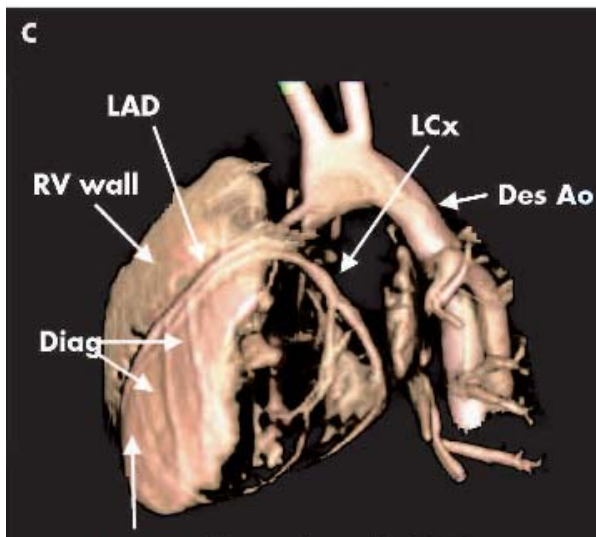

Anterior and apical wall of LV
D

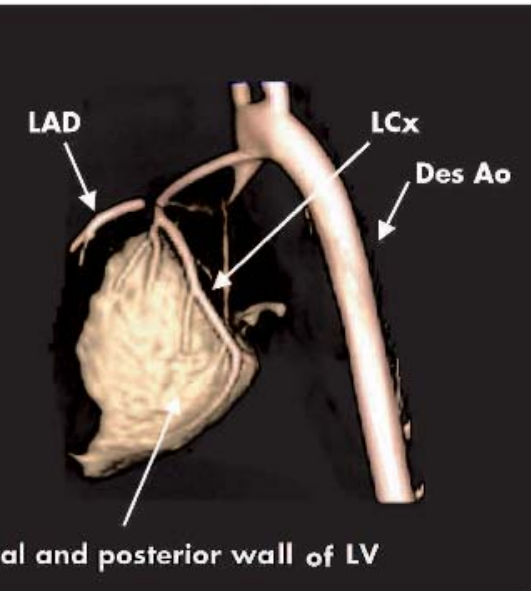

Figure 1 Volume rendered reconstruction images of porcine hearts using enhanced 256 slice high speed cone beam computed tomography (CT) acquired 5 seconds after injection of contrast material from the anterior view $(A, B)$ and the left anterior view $(C, D)$ revealed only the anterior, the apical interventricular septum (IVS) portion of the left ventricle (LV) myocardium, and the anterior wall of right ventricular myocardium supplied exclusively by the left anterior descending coronary artery (LAD) in pig 1 with LAD injection $(A, C)$, and the lateral and posterior portion of LV myocardium by the left circumflex artery $(\mathrm{LCX})$ in pig 2 with $L C x$ injection $(B, D)$. The right coronary artery was visualised from the backflow from the catheter located in the LAD in pig 1(A) as well as the aorta. Asc Ao, ascending aorta; Des Ao, descending aorta; Diag; diagonal branch.
LAD injection dimensional perfusion of the whole myocardium noninvasively, which is not possible with 16 slice CT. Using this technique, continuous 25 second acquisitions can be performed yielding quantitative myocardial perfusion information by using time density curves of myocardium on three dimensional images.

Especially using the former plan, this technique provides useful information for a physician to arrive at a strategy for percutaneous coronary intervention or coronary artery bypass surgery by combination of this new CT with selective intracoronary contrast injection. Furthermore, during PTSMA in subjects with hypertrophic obstructive cardiomyopathy, information regarding the septal branch of coronary arteries supplying the hypertrophic IVS which occludes the LV outflow tract is required. ${ }^{2}$ MCE with intracoronary contrast injection is commonly used during the procedure to guide the selection of the appropriate septal branch that feeds the hypertrophic IVS. As an alternative to MCE, the injection of contrast material from the catheter located in the septal branch followed by 1 second scanning using this CT technique can provide the proper information to select the appropriate septal branch; this technique is less complicated than MCE.

We described here a novel prototype CT that has the advantage of whole heart imaging in a single gantry rotation without ECG gated acquisition.

Of course, further research and technologic developments would be needed to overcome potential problems, such as radiation dose and any technical problems such as using the catheter without complication. Future technological advances in this area should overcome these problems. This technique may facilitate new cardiovascular diagnoses and may also find applications in other organs such as brain, liver, kidney, and vasculature.

\section{ACKNOWLEDGEMENTS}

This work was supported in part by the $4 \mathrm{D}-\mathrm{CT}$ research group at the National Institute of Radiological Sciences, Japan.

\section{Authors' affiliations}

N Funabashi, K Yoshida, K Nakagawa, N Komiyama, I Komuro, Department of Cardiovascular Science and Medicine, Chiba University Graduate School of Medicine, Chiba, Japan

H Tadokoro, K Odaka, T Tsunoo, S Mori, M Endo, S Tanada, National Institute of Radiological Sciences, Chiba, Japan

Correspondence to: Nobusada Funabashi, MD, Department of Cardiovascular Science and Medicine, Chiba University Graduate School of Medicine, 1-8-1 Inohana, Chuo-ku, Chiba City, Chiba 2608670, Japan; nobusada@ma.kcom.ne.jp

Accepted 27 January 2005

\section{REFERENCES}

1 Ito $\mathrm{H}$, Tomooka T, Sakai N, et al. Lack of myocardial perfusion immediately after successful thrombolysis: a predictor of poor recovery of left ventricular function in anterior myocardial infarction. Circulation 1992;85:1699-705.

2 van Dockum WG, ten Cate FJ, ten Berg JM, et al. Myocardial infarction after percutaneous transluminal septal myocardial ablation in hypertrophic 
obstructive cardiomyopathy: evaluation by contrast-enhanced magnetic resonance imaging. J Am Coll Cardiol 2004;43:27-34

3 Endo M, Tsunoo T, Kandatsu S, et al. Four-dimensional computed tomography (4D CT) concepts and preliminary development. Radiat Med 2003;21:17-22
4 Mori S, Endo M Tsunoo $T$, et al. Physical performance evaluation of a 256 slice CT scanner for four-dimensional imaging. Med Phys 2004;31:1348-56. 5 Leitz W, Axelsson B Szendrö G. Computed tomography dose assessment-A practical approach. Radiat Prot Dosimetry 1995;57:377-80.

\section{IMAGES IN CARDIOLOGY}

Transoesophageal echocardiography showing a thrombosis of the aortic valve mechanical replacement before and after thrombolytic treatment

\begin{abstract}
A 75 year old female patient with a four year history of aortic valve replacement with mechanical prosthesis (Medtronic Hall 20) was admitted to the cardiology department with a one month history of progressing dyspnoea and coughing episodes. She denied having chest pain. Admission ECG showed signs of hypertrophy and overload of the left ventricle and $1 \mathrm{~mm}$ ST segment elevations in leads II, III, and aVF. Routine laboratory tests revealed significant increases in cardiospecific enzymes and an international normalised ratio (INR) of 1.71, confirming inadequate anticoagulation treatment with long term administration of warfarin sodium.

Further management was strongly influenced by echocardiography findings. Transoesophageal echocardiography (TOE) on the day of admission revealed a non-dilated hypertrophic left ventricle (LV) with a hypokinetic inferior wall and good global ejection fraction (60\%). Mechanical disc prosthesis was present in the aortic position, with severe dysfunction. The whole right coronary sinus was filled with a hyperechogenic mass that hindered the movement of the disc and perfusion of the right coronary artery (panels A, B). Significant aortic stenosis was present with a peak gradient
\end{abstract}
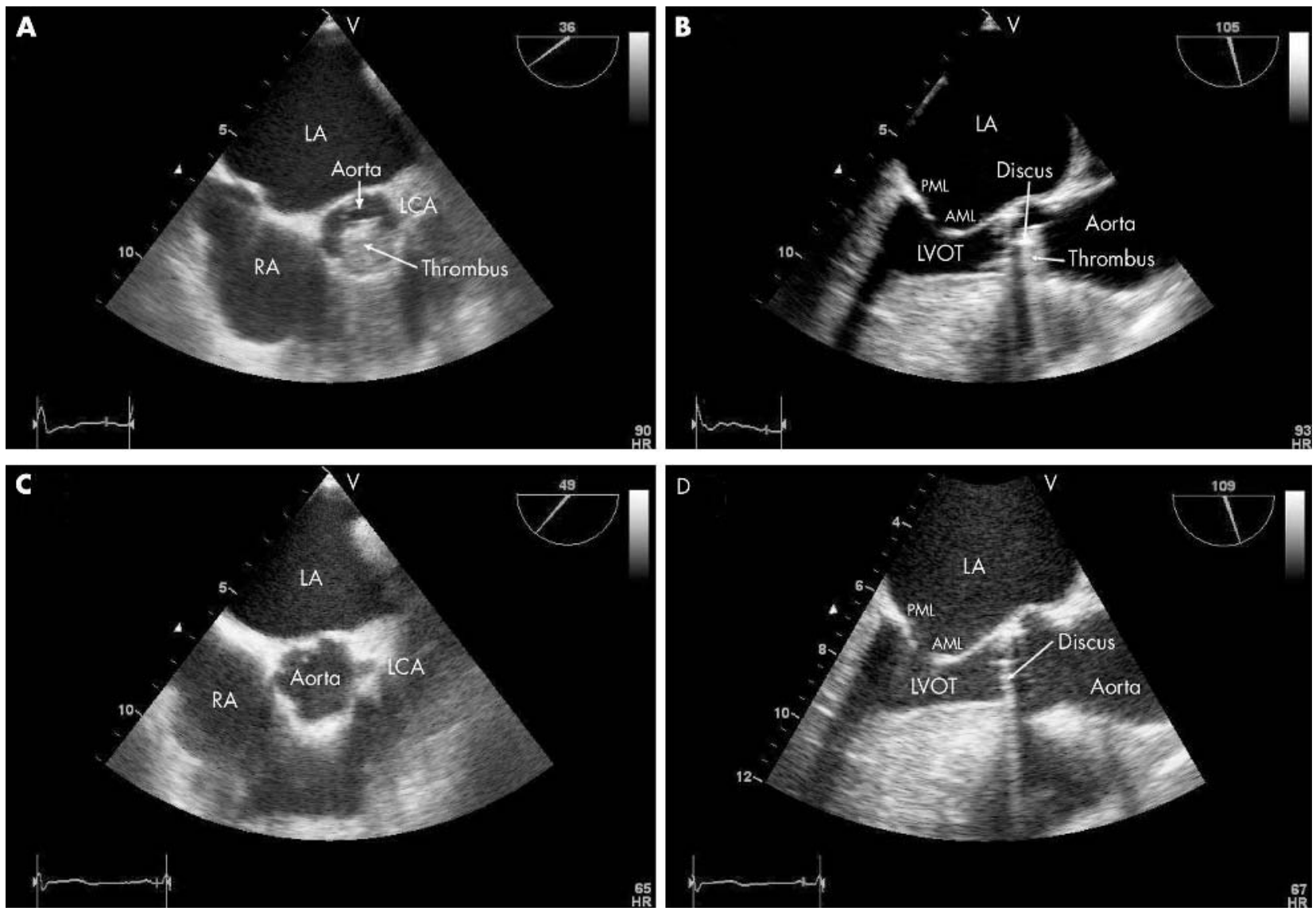

of $95 \mathrm{~mm} \mathrm{Hg}$ along with grade 2 regurgitation. Because of the high risk of surgery due to the ongoing myocardial infarction we decided to administer a thrombolytic agent. Actilyse $100 \mathrm{mg}$ was given intravenously in a two hour continual infusion, followed by a continuous infusion of unfractioned heparin during the following days.

A follow up TOE on day 5 after admission revealed a completely clear aortic bulb with no pathological masses, good function of the aortic mechanical valve, full motion range of the disc without any limitations, and a significant reduction of the peak grade down to 51-52 mm Hg, which was the patient's pre-morbid value (panels C, D). Global left ventricular function had no significant abnormalities. Anticoagulation treatment with warfarin sodium was resumed and the patient was discharged home on day 11 in satisfactory condition with an INR of 3.30.

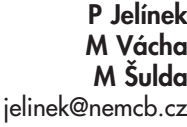

Jelínek

Šulda

jelinek@nemcb.cz 